\title{
Estimating dehalogenation reactivity of nanoscale zero-valent iron by simple colorimetric assay by way of 4-chlorophenol reduction
}

\author{
Paul D. Mines ${ }^{1,2}$, Kamilla M.S. Kaarsholm ${ }^{1}$, Ariadni Droumpali ${ }^{1}$, Henrik R. Andersen ${ }^{1}$, \\ Yuhoon Hwang ${ }^{3^{\dagger}}$ \\ ${ }^{1}$ Department of Environmental Engineering, Technical University of Denmark, Bygningstorvet, Denmark \\ ${ }^{2}$ Department of Micro- and Nanotechnology, Technical University of Denmark, Ørsteds Plads, Denmark \\ ${ }^{3}$ Department of Environmental Engineering, Seoul National University of Science and Technology, Seoul 01811, Republic of Korea
}

\begin{abstract}
A number of different nanoscale zero-valent iron (nZVI) materials have been prepared and compared depending on the desired properties for the particular application, but different physicochemical properties of this prepared nZVI make it difficult to universally compare and standardize them to the same scale. In this study, we aimed to demonstrate a simple microplate-based colorimetric assay using 4-chlorophenol as an indicator with respect to the remediation of real treatment targets, such as trichloroethylene (TCE), 1,1,1-trichloroethane (TCA), and atrazine. Effect of nickel contents on 4-chlorophenol reduction was successfully investigated by the miniaturized colorimetric assay. In the same manner, the effect of nickel contents on dehalogenation of TCE, TCA, and atrazine was investigated and the pseudo-first-order kinetic constants were compared with the results for 4-chlorophenol. The similar pattern could be observed between 4-chlorophenol reduction obtained by colorimetric assay and TCE, TCA, atrazine reduction obtained by a traditional chromatographic method. The reaction kinetics does not match perfectly, but the degree of reaction can be estimated. Therefore, the colorimetric assay can be a useful and simple screening tool to determine nZVI reactivity toward halogenated organics before it is applied to a particular remediation site.
\end{abstract}

Keywords: Colorimetric assay, Dehalogenation, Nanoscale zero-valent iron, Trichloroethylene, 4-chlorophenol

\section{Introduction}

Chlorinated solvents (e.g., solvents, pesticides) are widespread groundwater pollutants. They are among the most common pollutants at industrial sites due to their extensive application in chemicals production, metal degreasing, and dry cleaning. These compounds are environmentally persistent and may pose serious health threats due to their toxic and sometimes carcinogenic effects [1, 2]. Among $>342,000$ contaminated sites in the EU, around $10 \%$ is reported to be contaminated by chlorinated hydrocarbons [3]. In another report, chlorinated solvents were detected in 17\% (881 of 5,068 ) of the samples of groundwater in the USA. [4].

In this study, we selected trichloroethylene (TCE), 1,1,1-trichloroethane (TCA), and atrazine as the representative chlorinated organic treatment targets for nanoscale zero-valent iron (nZVI) application in soil and groundwater remediation. TCE and TCA are commonly used as an industrial solvent while atrazine is a herbicide of the triazine class. The maximum contaminant level (MCL) in the drinking water for those compounds was reported as $5 \mu \mathrm{g} / \mathrm{L}$ for TCE, $200 \mu \mathrm{g} / \mathrm{L}$ for TCA, and $3 \mu \mathrm{g} / \mathrm{L}$ for atrazine.

Approaches applied to the remediation of TCE and other DNAPLs include bioremediation, electrokinetics, flushing technologies (cosolvent/alcohol flooding, surfactant flushing), in situ oxidation, monitored natural attenuation, phytoremediation, (steam injection, electrical heating, in situ vitrification), volatilization technologies (soil vapor extraction, air sparging, in-well stripping), and treatment walls. nZVI has been applied to remediate those halogenated compounds based on reduction by elemental iron of halogenated
This is an Open Access article distributed under the terms of the Creative Commons Attribution Non-Commercial License (http://creativecommons.org/licenses/by-nc/3.0/) which permits unrestricted non-commercial use, distribution, and reproduction in any medium, provided the original work is properly cited.

Copyright (C) 2020 Korean Society of Environmental Engineers
Received January 11, 2019 Accepted March 7, 2019

${ }^{\dagger}$ Corresponding author

Email: yhhwang@seoultech.ac.kr

Tel: +82-2-970-6626 Fax: +82-2-971-5776

ORCID: 0000-0002-4013-3989 


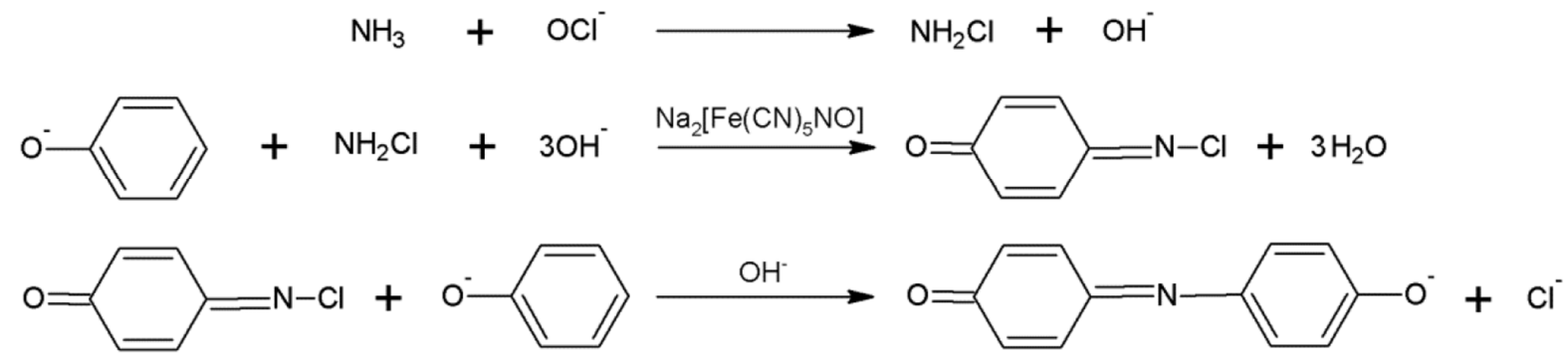

Fig. 1. Simplified reaction scheme of the modified indophenol reaction.

compounds. Metallic iron easily acts as an electron donor, and the electrons are in turn accepted by organically bound chlorine undergoing reductive dechlorination to form chloride. The reaction is energetically favorable as the standard reduction potential of ZVI $\left(\mathrm{Fe}^{2+} / \mathrm{Fe}\right)$ is $-0.44 \mathrm{~V}$, which is lower than many chlorinated organics [5]. The dehalogenation of halogenated organic compounds by nZVI is well demonstrated in laboratories as well as field studies [6-8]. In particular, many studies of halogenated organics degradation by nZVI have focused on TCE [9-11]. Although it is a slightly complicated series of reactions, the overall reaction can be represented stoichiometrically as Eq. (1) [11]:

$$
\begin{gathered}
\mathrm{HClC}=\mathrm{CCl}_{2}+4 \mathrm{Fe}^{0}+5 \mathrm{H}_{2} \mathrm{O} \longrightarrow \\
\mathrm{H}_{3} \mathrm{C}^{-} \mathrm{CH}_{3}+4 \mathrm{Fe}^{2+}+5 \mathrm{OH}^{-}+3 \mathrm{Cl}^{-}
\end{gathered}
$$

Depending on the application method of nZVI, different physical and chemical properties are required for successful application. For example, high mobility in subsurface water is possibly the most important feature for in-situ remediations using nZVI maintained in suspension. On the other hand, permeation of nZVI into treated water should be minimized by immobilizing nZVI in/on to a supporting media for ex-situ application. In order to obtain the desired properties for the particular application, a number of synthesis and modification methods have been reported in past decades. For in-situ applications where colloidal stability in the subsurface is necessary, surface modification of nZVI has been applied by using various surface stabilizing agents, such as surfactants, polymers, and organic clays [12-15]. While for ex-situ applications where particle immobilization is mandatory, attachment of nZVI on to supporting media has been extensively studied using various materials, such as alginate beads, activated carbon, and polymeric membranes [16-19].

Although a number of different nZVI materials have been prepared and compared, across many intensive studies, different physicochemical properties of these prepared nZVI's make it quite difficult to universally compare and standardize them to the same scale. Depending on the aim of research and laboratory environment, the synthesis conditions of nZVI can be significantly varied; and, with that comes potentially considerably different nZVI properties. Moreover, nZVI is inherently oxidized by reaction with not only oxygen but also water and the dissolved oxygen in the water; which bring about different chemical properties of nZVI with respect to time $[20,21]$. It is apparent that there are a plethora of factors, ranging from synthesis conditions to local environmental conditions, to storage and subsequent target delivery conditions, which can change the properties unique to each batch of nZVI. Therefore, one of the main concerns in the study and application of nZVI is how to measure its reductive reactivity, both easily and quickly.

In this light, in our previous work [22] we developed a simple assay to characterize the reductive reactivity of nZVI using 4-chlorophenol as the substrate; then, quantifying the reduction product, phenol, using a slightly modified version of the well-known indophenol (Fig. 1) colorimetric reaction [23].

By utilizing a straightforward and user-friendly colorimetric assay, it is thus possible to avoid time-consuming and expensive chromatographic equipment. This assay was further miniaturized into microplate format, allowing for rapid analysis if multiple samples and drastically reducing reagent and sample volumes [24]. The applicability of the developed assay was well demonstrated with both bare nZVI and bimetallic Ni/Fe-NPs. As it was that dehalogenation was the targeted reduction mechanism, 4-chlorophenol was selected as an indicator due to its similarity with targeted chlorinated compounds. However, there is lack of verification on the effectiveness and applicability of the suggested colorimetric assay using 4-chlorophenol as an indicator with respect to the remediation of real treatment targets, such as chlorinated solvents and pesticides by nZVI.

In this study, we selected TCE, TCA, and atrazine as the representative chlorinated organic treatment targets for nZVI application in soil and groundwater remediation. Dehalogenation batch tests for TCE, TCA, and atrazine were performed using bimetallic $\mathrm{Ni} / \mathrm{Fe}-\mathrm{NPs}$. The concentration of each treatment target was monitored by either traditional gas chromatography-mass spectrometry (GC-MS) equipped with a purge-and-trap concentrator or high-performance liquid chromatography (HPLC). The detailed analytical method together with the developed reaction termination method used was that in our previously reported work [25]. Finally, the overall results are compared with the results from the colorimetric assay to confirm the ability of the colorimetric assay to describe the reductive reactivity of nZVI. Therefore, the research objective is to verify the effectiveness of the colorimetric assay to be used as a screening method prior to applying for real treatment targets in order to save time and effort.

\section{Materials and Methods}

\subsection{Chemicals}

Iron(II) sulfate heptahydrate $\left(\mathrm{FeSO}_{4} \cdot 7 \mathrm{H}_{2} \mathrm{O},>99 \%\right.$, Sigma-Aldrich), 
nickel sulfate hexahydrate $\left(\mathrm{NiSO}_{4} \cdot 6 \mathrm{H}_{2} \mathrm{O},>99 \%\right.$, Sigma-Aldrich), sodium borohydride $\left(\mathrm{NaBH}_{4},>98 \%\right.$, Sigma-Aldrich) and ethanol (> 99.9\%, Sigma-Aldrich) were used for nZVI synthesis. Trichloroethylene (TCE, > 99.5\%, Sigma-Aldrich), 1,1,1-trichloroethane (TCA, $5,000 \mu \mathrm{g} / \mathrm{mL}$ in methanol, Sigma-Aldrich), atrazine (PESTANAL $^{\circledR}, \quad 98.8 \%$, Sigma-Aldrich), phenol (> 99\%, Sigma-Aldrich), 4-chlorophenol (> 98\%, Merck), and Sodium bromate $\left(\mathrm{NaBrO}_{3},>99 \%\right.$, Sigma-Aldrich), sodium carbonate $\left(\mathrm{Na}_{2} \mathrm{CO}_{3}\right.$, $>99 \%$, Sigma-Aldrich) were used for reactivity test. Sodium hypochlorite solution (NaClO, 10\%, Sigma-Aldrich), ammonium chloride $\left(\mathrm{NH}_{4} \mathrm{Cl},>99.5 \%\right.$, Sigma-Aldrich), sodium nitroprusside $\left(\mathrm{Na}_{2}\left[\mathrm{Fe}(\mathrm{CN})_{5} \mathrm{NO}\right] \cdot 2 \mathrm{H}_{2} \mathrm{O},>99 \%\right.$, Merck), sodium hydroxide $(\mathrm{NaOH}$, $>98 \%$, Sigma-Aldrich) were used for the modified indophenol reaction. Unless otherwise stated, all chemicals were used, as obtained, from the supplier.

\subsection{Preparation of Bimetallic Ni/Fe-NPs}

The preparation of nZVI for this study utilized the mild chemical reduction of metal salts in the solution phase, as was used in the previous study regarding the method development for nZVI reaction termination [25], and is outlined below in Eq. (2):

$$
2 \mathrm{Fe}^{2+}+\mathrm{BH}_{4}+3 \mathrm{H}_{2} \mathrm{O} \rightarrow 2 \mathrm{Fe}^{0}+\mathrm{H}_{2} \mathrm{BO}_{3}^{-}+4 \mathrm{H}^{+}+2 \mathrm{H}_{2} \uparrow
$$

Synthesis of nZVI was conducted in a 1,000 mL three-neck round-bottom flask reactor, having the central neck fitted with a tunable mechanical stirrer; the two remaining necks were sealed with rubber septa. The iron precursor solution was prepared with $600 \mathrm{~mL}$ of $0.24 \mathrm{M} \mathrm{FeSO}_{4} \cdot 7 \mathrm{H}_{2} \mathrm{O}$ in deionized water, and the reduction of the iron was initiated by introducing $200 \mathrm{~mL}$ of $1.8 \mathrm{M} \mathrm{NaBH}_{4}$ solution in deionized water via a peristaltic pump, with a constant delivery rate of $10 \mathrm{~mL} / \mathrm{min}$. Collection and washing of the nZVI were performed via centrifugation $(2,500 \mathrm{RPM}, 5 \mathrm{~min}$, Heraeus Multifuge X1, Thermo Scientific, USA), and the collected pellet of nZVI was then washed once with deionized water and twice with ethanol, interspersed with centrifugation. Once washed, the nZVI particles were dried in a vacuum oven at $60^{\circ} \mathrm{C}$ for $4 \mathrm{~h}$ and subsequently stored in air-tight vials inside an anaerobic chamber $\left(\mathrm{N}_{2}: \mathrm{H}_{2}=95: 5\right.$, Coy Laboratory Products, USA), to avoid any oxidation. The physical characteristics of the prepared nZVI are considered to be identical to uncoated nZVI prepared in the previously mentioned study [20], as the same synthesis protocol was used.

Ni-doped bimetallic nZVI particles (Ni/Fe-NPs) were prepared via chemical reduction, as presented in the previously mentioned study [25]. The secondary metal solution $\left(\mathrm{NiSO}_{4}\right)$ was introduced to the nZVI particles, whereupon bimetallic Ni/Fe is inherently generated by the attachment of nickel on the iron surface. The mixture was placed in a sonication bath for $3 \mathrm{~min}$, followed by $10 \mathrm{~min}$ of vortex mixing, in order to completely disperse the particles and minimize any aggregates that may have been formed throughout the process. All mixing was conducted in an anaerobic chamber, and the vials were capped securely with a PTFE/silicone septum and aluminum crimp seal, to avoid any oxidation. After $10 \mathrm{~min}$ of redox reaction between the nickel and the nZVI, the resulting $\mathrm{Ni} / \mathrm{Fe}-\mathrm{NPs}$ were collected and washed twice with deionized water and centrifugation. Prepared Ni/Fe-NPs were immediately applied in the reactivity tests; therefore, further drying was not needed or considered.

\subsection{Characterization of $\mathrm{Ni} / \mathrm{Fe}$ Nanoparticles (Ni/Fe-NPs)}

Surface imaging and morphology of the prepared Ni/Fe-NPs was performed using field emission-transmission electron microscopy (FE-TEM, FEI, Tecnai T20 G', and The Netherlands). Additionally, elemental mapping of the particles was performed using the scanning transmission electron microscopy (TEM) feature on the TEM instrument, coupled with energy dispersive X-ray spectroscopy (STEM-EDS). Data treatment for the STEM-EDS was analyzed with Aztec software (Oxford Instruments, UK). TEM analysis was conducted by sonicating and applying a droplet of Ni/Fe-NPs suspended in ethanol on to a carbon-coated copper mesh grid (Formvar Carbon Film on 300 Mesh Copper Grid, Agar Scientific, United Kingdom).

\subsection{Dehalogenation Test}

Reactivity of bimetallic Ni/Fe-NPs were tested with various groundwater contaminants: TCE, TCA, atrazine, and 4-chlorophenol. As prepared solutions of $\mathrm{Ni} / \mathrm{Fe}-\mathrm{NPs}$, as mentioned in section 2.2, were re-suspended in $50 \mathrm{~mL}$ of fresh deionized water, along with three glass beads (diameter $=4 \mathrm{~mm}$ ) to ensure turbulent conditions and adequate mixing during reactivity testing. The concentration of bimetallic Ni/Fe-NPs was determined as $2 \mathrm{~g} / \mathrm{L}$. The vials were then immediately spiked with stock solutions of TCE, TCA, atrazine, or 4-chlorophenol (in separate vials). In order to simulate realistic conditions, a concentration of $300 \mu \mathrm{g} / \mathrm{L}$ of TCE and TCA was assessed, as that is slightly higher than reported values (i.e., $\sim 250$ $\mu \mathrm{g} / \mathrm{L}$ ) for TCE detected at contaminated sites in Danish groundwater [26]. In the case of 4-chlorophenol, the initial concentration was determined to be $500 \mu \mathrm{M}$, which is identical to the previously mentioned study regarding the colorimetric assay [24]. Initial atrazine concentration was determined to be $120 \mu \mathrm{g} / \mathrm{L}$, as that concentration is slightly higher than values (i.e., 100 $\mu \mathrm{g} / \mathrm{L}$ ) observed in the past for various contaminated river waters in the USA [27].

At the desired sampling time, sodium bromate stock solution was added into the reaction vials to terminate the reactivity of the remaining Ni/Fe-NPs (Eq. (3)), as described in our previous study in detail [25]. The concentration of bromate was determined to be double the stoichiometric amount needed for complete reaction with the nZVI in the reactor; therefore, $23.9 \mathrm{mM}$ [28].

$$
\mathrm{BrO}_{3}^{-}+3 \mathrm{Fe}^{0}+3 \mathrm{H}_{2} \mathrm{O} \rightarrow \mathrm{Br}^{-}+3 \mathrm{Fe}^{2+}+6 \mathrm{OH}^{-}
$$

\subsection{Analysis}

\subsubsection{TCE and TCA}

Samples were analyzed for TCE and TCA using the purge-and-trap technique (purge temperature $=30^{\circ} \mathrm{C}$, Velocity XPT Purge-and-Trap Sample Concentrator, AQUATek 70 auto-sampler, Vocarb ${ }^{\circledR} 3000$ Trap, Teledyne Tekmar, OH, USA) coupled with gas chromatography-mass spectrometry (GC-MS, HP 6890 Series GC System, 5973 Mass Selective Detector, Hewlett Packard, CA, USA). Compound separation in the GC-MS was performed with a fused silica capillary column (length $=30.0 \mathrm{~m}$, inside diameter $=0.25 \mathrm{~mm}$, film thickness 
$=1.5 \mu \mathrm{m}$, VOCOL $^{\circledR}$, Supelco, Sigma-Aldrich, MO, USA). All apparatus settings were used per a previous study investigating trihalomethanes and other disinfection by-products in swimming pool water [29]. However, different ion ratios for quality control were used for quantification by mass spectrometry. For TCE, the quantifier and qualifier ion ratios were: $\mathrm{m} / \mathrm{Z}=130$ and $\mathrm{m} / \mathrm{Z}=95$, respectively. For TCA, the quantifier and qualifier ion ratios used were: $\mathrm{m} / \mathrm{Z}=97$ and $\mathrm{m} / \mathrm{Z}=61$, respectively. Calibration curves were prepared in the range of $0.3-300 \mu \mathrm{g} / \mathrm{L}$, and the quantification limit was set to $0.3 \mu \mathrm{g} / \mathrm{L}$, based on the lowest standard used.

\subsubsection{Atrazine}

Samples were analyzed for atrazine using HPLC (Dionex UltiMate 3000 UHPLC; with a Multiple Wavelength Detector, MWD-3000RS, Thermo Scientific, USA). Atrazine was separated on a C18 reversed-phase column (Hypersil BDS C18, $5 \mu \mathrm{m}, 150 \times 4.6 \mathrm{~mm}$, Thermo Scientific, USA) equipped with a guard column (Hypersil BDS C18, $5 \mu \mathrm{m}, 10 \times 4.0 \mathrm{~mm}$, Thermo Scientific, USA). Separation was achieved with an eluent gradient of acetonitrile and deionized water, the flow rate was kept constant at $1.5 \mathrm{~mL} / \mathrm{min}$, the injection volume was $45 \mu \mathrm{L}$, the column temperature was kept at $30^{\circ} \mathrm{C}$, and the total analysis time was $10 \mathrm{~min}$ per sample, including equilibration time. Detection and quantification were done with a UV detector at a wavelength of $210 \mathrm{~nm}$. Calibration curves were prepared in the range of $10-300 \mu \mathrm{g} / \mathrm{L}$, and the quantification limit was set to $10 \mu \mathrm{g} / \mathrm{L}$, based on the lowest standard used.

\subsubsection{4-chlorophenol}

In order to obtain the 4-chlorophenol concentration in the reaction vials, the reaction product, phenol, was measured by the colorimetric assay developed in our previous study [22]; using the reaction with chloramine to form the blue indophenol dye (Fig. 1). In that study, phenol detection based on the indophenol reaction was successfully developed by using phenol as the limiting reactant in the overall reaction. Briefly, reagent A was prepared by diluting $0.2 \mathrm{~mL}$ of $10 \%$ hypochlorite solution and $66 \mathrm{~mL}$ of $0.5 \mathrm{M} \mathrm{NaOH}$ solution to $100 \mathrm{~mL}$ with deionized water; and, reagent B was prepared as $100 \mathrm{mg}-\mathrm{NH}_{4}-\mathrm{N} / \mathrm{L}$ of ammonium chloride and $0.3 \mathrm{~g} / \mathrm{L}$ of sodium nitroprusside in deionized water. A drop of hydrochloric acid was added to reagent $\mathrm{B}$ to lower the $\mathrm{pH}$ and minimize ammonia stripping into the headspace of the reaction vial. Equal volumes of reagents $\mathrm{A}$ and $\mathrm{B}$ were pre-mixed to make the reagent $\mathrm{AB}$. Then, $2.3 \mathrm{~mL}$ of the analysis sample was transferred into a cuvette, and $0.2 \mathrm{~mL}$ of reagent $\mathrm{AB}$ was added to the sample. The mixture was allowed to react in the dark for $2 \mathrm{~h}$ and was subsequently quantified by measuring the absorbance with a UV-vis spectrometer (AAnalyst 200, Perkin-Elmer, USA) at $680 \mathrm{~nm}$.

\section{Results and Discussion}

\subsection{Morphology of Prepared Bimetallic Ni/Fe-NP}

The morphologies of the Ni/Fe-NPs prepared in this study were observed using TEM and are pictured in Fig. 2(a) and 2(b) with different magnifications. The Ni/Fe-NPs were spherical and were aggregated together in a chain-like network. This spherical shape, chain net morphology, and appearance of the iron-oxide shell surrounding the nZVI core were similar to those observed in other research works [30-32] studying nZVI particles. Particle sizes ranged in diameters between 25 to $250 \mathrm{~nm}$, with the bulk of the particles falling in the $60-90 \mathrm{~nm}$ range. The bulk of the Ni/Fe-NPs also possessed an iron-oxide shell with thicknesses ranging from 3 to $6 \mathrm{~nm}$. Determination of nickel on the nZVI surface is extremely difficult with normal observation with TEM, therefore scanning-TEM coupled with energy dispersive X-ray spectroscopy (STEM-EDS) was employed to visually identify "islands" of nickel found on the surface of the nZVI. Although the quality of the image decreases when using STEM, it is clearly visible with the EDS coupling that islands of nickel (in green) do appear on the surface of the nZVI particles (in red), pictured in Fig. 2(c) (STEM-EDS). The observed particles, including the STEM-EDS mapping of iron and nickel, are identical to those observed in the previously mentioned study regarding nZVI reactivity termination that synthesized particles following the same protocol [25]. Furthermore, although not completely reliable for overall quantita-
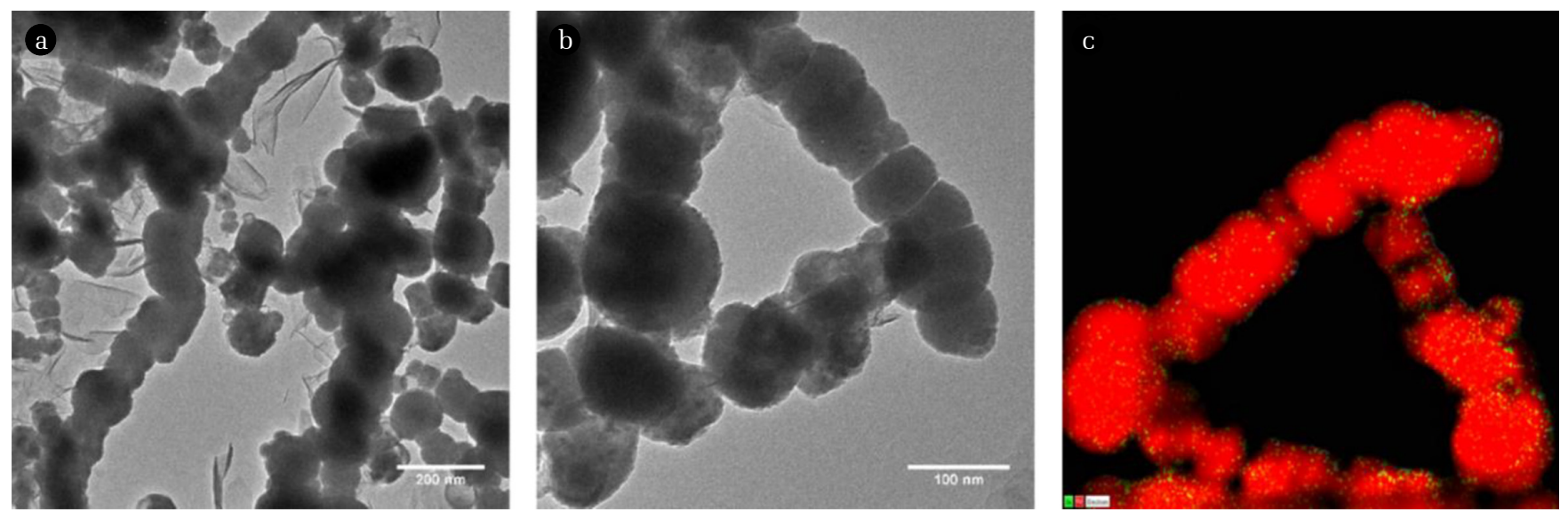

Fig. 2. Transmission electron microscope imaging of Ni/Fe-NPs: a) TEM at lower magnification; b) TEM at higher magnification; and c) STEM-EDS mapping of image in b (rotated for analysis within instrument) with iron (visualized in red) as the dominant species with "islands" of nickel (visualized in green) blanketing the particles. 
tive elemental analysis, the EDS spectrum plot generated during mapping correlated similar nickel amounts with respect to iron; identifying $4.2 \%$ nickel in $5 \% \mathrm{Ni} / \mathrm{Fe}-\mathrm{NPs}$.

\subsection{Colorimetric Assay for 4-chlorophenol Dehalogenation}

The previously developed miniaturized colorimetric assay for 4-chlorophenol dehalogenation was used to investigate the effect of nickel content on the dehalogenation kinetics of 4-chlorophenol by nZVI. Nickel content was varied over the range of $0.01-5 \%$; and, pseudo-first-order kinetics were applied to interpret the results; of which the calculated kinetics constants are presented in Fig. 3. As expected, higher nickel content generally brings about a faster reaction when it is coupled with iron and other metals in the form of a catalytic alloy [33]. However, after approximately $1 \%$ (wt.) nickel concentration on the particles, the net return in reactivity increase is lower. The idea behind this is that as the nickel loading increases, the contact area between the two metals decreases and there is a loss in positive effects with respect to reactive hydrogen generation, electron transport, catalytic sites, and galvanic cell formation. Similar findings have been observed before with secondary metal doped nZVI for dechlorination [34, 35] and nitrophenol reduction [36].

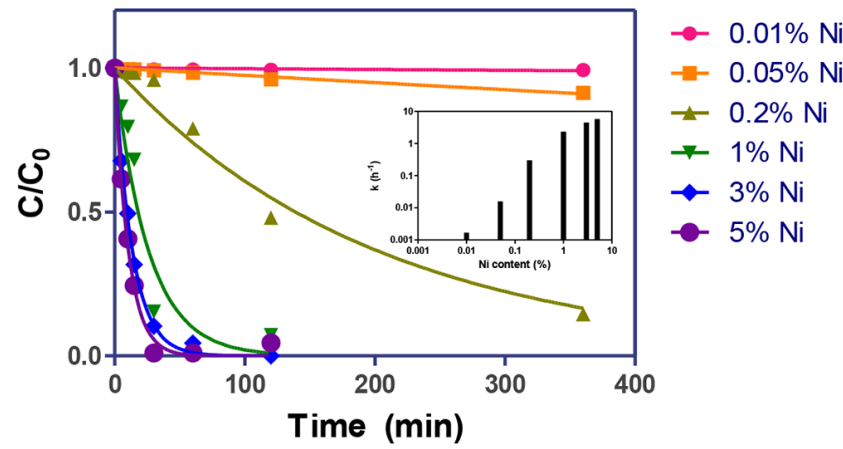

Fig. 3. Effect of nickel content on 4-chlorophenol reduction by $\mathrm{nZVI}$. Derived rate constants by curve-fitting are plotted on a log10-scale in the inset. Note that the curves are fitted with pseudo-first-order kinetics. Figure adapted from our previous work [24].

\subsection{Dehalogenation of TCE, TCA, and Atrazine by Ni/Fe-NPs}

\subsubsection{Role of nickel for dehalogenation process}

The previous section 3.2 describes the use of the colorimetric assay developed in our research group previously [24]. It was effective to determine the catalytic reactivity of $\mathrm{Ni} / \mathrm{Fe}-\mathrm{NPs}$ in a simple manner, without complicated analytical equipment and a trained technician. However, there is still an issue about the reliability of measured reaction rate constants. Many typical treatment targets for nZVI and its bimetallic amalgamated counterparts are halogenated organic groundwater contaminants, such as TCE and TCA. As it is that the colorimetric assay obtains results only for 4-chlorophenol, rather than actual water treatment targets, it should be verified that the results for 4-chlorophenol dehalogenation obtained by the colorimetric assay have a positive relationship with the results for actual treatment target decontamination obtained by traditional analytical methods, such as GC-MS or HPLC.
As a first step, we investigated the role of nickel on TCE, TCA, and atrazine dehalogenation. It is hypothesized that the dehalogenation mechanism of the tested compounds (i.e., TCE, TCA, atrazine, and 4-chlorophenol) are similar in terms of substituting the halogen by hydrogen through hydrogenesis catalysis, performed by nickel in this study. Therefore, it is expected that dehalogenation results will exhibit a similar pattern with nickel content, although the kinetics may be different. To investigate this, we started by looking at the role of nickel on the process, as well as adding acetate as a buffer to nZVI without nickel (Fig. 4). This was done with an initial TCE concentration of $300 \mu \mathrm{g} / \mathrm{L}$, adding particles at a dose of $2 \mathrm{~g} / \mathrm{L}$, and allowing them to react with constant mixing for $1 \mathrm{~h}$. The results for nZVI alone and nZVI buffered with acetate yielded no change in the TCE concentration; however, once nickel was added $(5 \% \mathrm{w} / \mathrm{w})$ to make $\mathrm{Ni} / \mathrm{Fe}-\mathrm{NPs}$, the results were very clear that the catalytic reaction causing dehalogenation was taking place. The TCE was nearly completely degraded, where only 0.07 $\mu \mathrm{g} / \mathrm{L}$ was detected after the $1 \mathrm{~h}$ reaction time. This result correlates identically with the results of 4-chlorophenol reduction described in the previous section. Meaning that there is evidence for the applicability of the described colorimetric assay to estimate the dehalogenation ability of nZVI materials on real-world contaminants. However, the data at this point does not allow for kinetics constants to be determined or provide for a full comparison to the colorimetric assay. This was done by changing the reaction conditions (i.e., lowering nickel concentrations) to decrease the reaction rate into a measurable range for adequate comparison. Again, the overall research objective is to comparing dehalogenation kinetics of various halogenated compounds, not to find optimal conditions for dehalogenation.

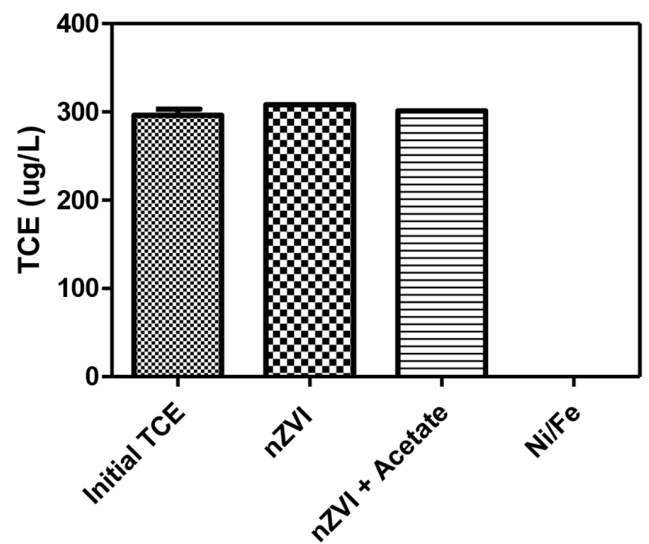

Fig. 4. Effect of buffer and nickel on TCE reduction by nZVI (Initial TCE concentration $=300 \mu \mathrm{g} / \mathrm{L}, \mathrm{nZVI}$ or $\mathrm{Ni} /$ Fe dosage $=2 \mathrm{~g} / \mathrm{L}$, acetate concentration $=10 \mathrm{mM}$, reaction time $=1 \mathrm{~h}$ )

\subsubsection{Effect of nickel contents on dehalogenation of TCE, TCA, and atrazine.}

In order to achieve slower kinetics in our experimental set-up, lowering the nickel loading on nZVI (i.e., 0.1, 0.2, and 0.5\%) was tested for the dehalogenation of TCE, TCA, and atrazine (Fig. 5). As expected, the higher nickel loadings increased the dehalogenation of each contaminant, with TCE (Fig. 5(a)) and TCA (Fig. 5(b)) 

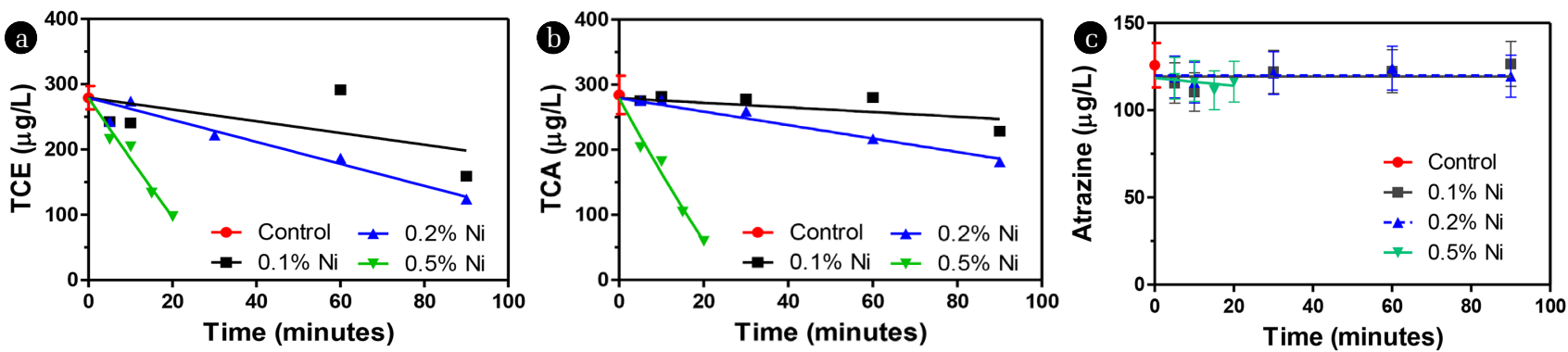

Fig. 5. Effect of nickel content on TCE (a), TCA (b), and atrazine (c) reduction by Ni/Fe-NPs. Curves are fitted using pseudo-first-order kinetics.

behaving quite similarly, while atrazine (Fig. 5(c)) had much lower reduction kinetics.

$\mathrm{Ni} / \mathrm{Fe}-\mathrm{NPs}$ with $0.1 \%$ nickel loading exhibited $42 \%$ of TCE reduction in 90 min, while more than 65\% TCE reduction could be achieved within just 20 min when there was $0.5 \%$ nickel loading. Similar results were also achieved when reducing TCA with $\mathrm{Ni} / \mathrm{Fe}-\mathrm{NPs}$ at various nickel loadings. However, atrazine demonstrated noticeable slower reaction kinetics compared to both TCE and TCA. There was effectively no atrazine reduction observed until there was a nickel loading of $0.2 \%$, but a slight decrease of atrazine concentration was produced by $0.5 \%$ nickel loading; while, ultimately fast atrazine reduction was obtained when the nickel loading was drastically increased to $5 \%$. These results are quite similar to the results previously described with 4-chlorophenol reduction. The cause of this can be attributed to hydrogenesis, but more specifically certain other phenomena have been proposed for the bimetallic nZVI degradation of compounds, such as $\beta$ -elimination [37, 38].

\subsection{Comparative Study for Reaction Kinetics}

The similar pattern could be observed between 4-chlorophenol reduction obtained by colorimetric assay and TCE, TCA, atrazine reduction obtained by a traditional chromatographic method, which makes the colorimetric assay as a simple tool to estimate overall performance of hydrogenation. However, the reaction kinetics seems to be significantly different from the halogenated compounds we tested, therefore, a more in-depth kinetics study was performed.

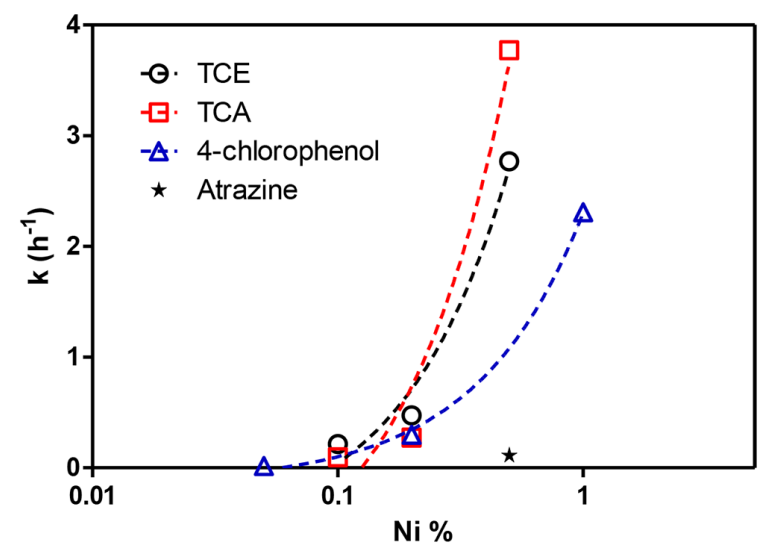

Fig. 6. Reaction rate constants of TCE, TCA, 4-chlorophenol, and atrazine with respect to nickel content.
Fig. 6 plots the kinetics constants calculated by pseudo-first order kinetics with respect to nickel content. Atrazine was not used for the kinetic analysis, because it was possible to obtain a kinetic constant only for $0.5 \%$ of nickel content. However, the kinetic constant obtained for $0.5 \%$ of nickel content was also presented in Fig. 5 for further comparison. Among the three compounds we compared, TCE, TCA, and 4-chlorophenol, 4-chlorophenol showed the slowest kinetics after atrazine. In the case of TCE and TCA, the kinetic is slower when the concentration is low compared to the linear line, but the reaction rate constant was significantly increased at $0.5 \%$ of nickel content. This indicates that there is a certain ratio between iron and nickel to maximize dehalogenation.

This result shows that the colorimetric assay using 4-chlorophenol as an indicating substrate can simulate dehalogenation reactivity of nZVI and its bimetallic product successfully, and it agrees well with other chlorinated organics. The reaction kinetics does not match perfectly, but the degree of reaction can be estimated. Moreover, 4-chlorophenol reduction kinetics is slower than real-world treatment targets TCE or TCA, which make 4-chlorophenol reduction an indicator for sufficient reductive reactivity toward TCE or TCA.

\section{Conclusions}

In this study, we verified the effectiveness of the colorimetric assay developed in our previous research for estimating dehalogenation reactivity of nZVI toward real treatment targets such as TCE, TCA, and atrazine. The previously developed miniaturized colorimetric assay for 4-chlorophenol dehalogenation was used to investigate the effect of nickel contents on the dehalogenation kinetics of 4-chlorophenol by nZVI. In the same manner, the effect of nickel contents on the dehalogenation kinetics of TCE, TCA, and atrazine was investigated by traditional chromatographic methods. Generally, higher nickel contents brought faster reduction although the reaction kinetics seems to be significantly different depending on the halogenated compounds. For example, TCA and TCE could be reduced by Ni/Fe-NPs with $0.1 \%$ nickel loading, while there was effectively no atrazine reduction observed until there was a nickel loading of $0.2 \%$. When the reaction kinetics was compared, 4-chlorophenol showed slower kinetics compared to TCE and TCA. The reaction kinetics does not match perfectly, but the degree of reaction can be estimated. Moreover, 4-chlorophenol reduction kinetics is slower than real-world treatment targets TCE or TCA, 
which make 4-chlorophenol reduction an indicator for sufficient reductive reactivity toward TCE or TCA. Therefore, the colorimetric assay can be a useful and simple screening tool to determine nZVI reactivity toward halogenated organics before it is applied to a particular remediation site.

\section{Acknowledgments}

This work is supported by a National Research Foundation of Korea (NRF) grant funded by the Korea government (Ministry of Science and ICT) (No. NRF-2017R1C1B5015387).

\section{References}

1. Henschler D. Toxicity of chlorinated organic compounds: Effects of the introduction of chlorine in organic molecules. Angew. Chem. Int. Ed. Engl. 1994;33:1920-1935.

2. Ruder AM. Potential health effects of occupational chlorinated solvent exposure. Ann. NY. Acad. Sci. 2006;1076:207-227.

3. Panagos P, Van Liedekerke M, Yigini Y, Montanarella L. Contaminated sites in Europe: Review of the current situation based on data collected through a European network. J. Environ. Public Health 2013;2013:158764.

4. Moran MJ, Zogorski JS, Squillace PJ. Chlorinated solvents in groundwater of the United States. Environ. Sci. Technol. 2007;41:74-81

5. Crane RA, Scott TB. Nanoscale zero-valent iron: Future prospects for an emerging water treatment technology. J. Hazard. Mater. 2012;211-212:112-125.

6. Liu Y, Majetich SA, Tilton RD, Sholl DS, Lowry GV. TCE dechlorination rates, pathways, and efficiency of nanoscale iron particles with different properties. Environ. Sci. Technol. 2005;39:1338-1345.

7. Mueller NC, Braun J, Bruns J, et al. Application of nanoscale zero valent iron (NZVI) for groundwater remediation in Europe. Environ. Sci. Pollut. Res. 2012;19:550-558.

8. Bae S, Lee W. Influence of riboflavin on nanoscale zero-valent iron reactivity during the degradation of carbon tetrachloride. Environ. Sci. Technol. 2014;48:2368-2376.

9. Bezbaruah AN, Thompson JM, Chisholm BJ. Remediation of alachlor and atrazine contaminated water with zero-valent iron nanoparticles. J. Environ. Sci. Health B. 2009;44:518-524.

10. Wang Q, Jeong SW, Choi H. Removal of trichloroethylene DNAPL trapped in porous media using nanoscale zerovalent iron and bimetallic nanoparticles: Direct observation and quantification. J. Hazard. Mater. 2012;213-214:299-310.

11. Li S, Fang Y, Romanczuk CD, Jin Z, Li T, Wong MS. Establishing the trichloroethene dechlorination rates of palladium-based catalysts and iron-based reductants. Appl. Catal. B-Environ. 2012;125:95-102.

12. Krol MM, Oleniuk AJ, Kocur CM, et al. A field-validated model for in situ transport of polymer-stabilized nZVI and implications for subsurface injection. Environ. Sci. Technol. 2013;47:7332-7340.

13. Hwang Y, Lee Y, Mines PD, Huh YS, Andersen HR. Nanoscale zero-valent iron (nZVI) synthesis in a Mg-aminoclay solution exhibits increased stability and reactivity for reductive decontamination. Appl. Catal. B-Environ. 2014;147:748-755.

14. Mines PD, Byun J, Hwang Y, Patel HA, Andersen HR, Yavuz CT. Nanoporous networks as effective stabilisation matrices for nanoscale zero-valent iron and groundwater pollutant removal. J. Mater. Chem. A. 2016;4:632-639.

15. Saberinasr A, Rezaei M, Nakhaei M, Hosseini SM. Transport of CMC-stabilized nZVI in saturated sand column: The effect of particle concentration and soil grain size. Water Air Soil Pollut. 2016;227:394.

16. Choi H, Al-Abed S, Agarwal S, Dionysiou DD. Synthesis of reactive nano-Fe/Pd bimetallic system-impregnated activated carbon for the simultaneous adsorption and dechlorination of PCBs. Chem. Mater. 2008;20:3649-3655.

17. Kim H, Hong HJ, Jung J, Kim SH, Yang JW. Degradation of trichloroethylene (TCE) by nanoscale zero-valent iron (nZVI) immobilized in alginate bead. J. Hazard. Mater. 2010;176: 1038-1043.

18. Liu C, Li X, Ma B, Qin A, He C. Removal of water contaminants by nanoscale zero-valent iron immobilized in PAN-based oxidized membrane. Appl. Surf. Sci. 2014;321:158-165.

19. Mines PD, Uthuppu B, Thirion D, et al. Granular activated carbon with grafted nanoporous polymer enhances nanoscale zero-valent iron impregnation and water contaminant removal. Chem. Eng. J. 2018;339:22-31.

20. Hwang Y, Lee Y, Mines PD, Oh Y, Choi JS, Andersen HR. Investigation of washing and storage strategy on aging of Mg-aminoclay (MgAC) coated nanoscale zero-valent iron (nZVI) particles. Chem. Eng. Sci. 2014;119:310-317.

21. Zhang Y, Chen W, Dai C, Zhou C, Zhou X. Structural evolution of nanoscale zero-valent iron (nZVI) in anoxic $\mathrm{Co}^{2+}$ solution: Interactional performance and mechanism. Sci. Rep. 2015;5:13966

22. Hwang Y, Mines PD, Jakobsen MH, Andersen HR. Simple colorimetric assay for dehalogenation reactivity of nanoscale zero-valent iron using 4-chlorophenol. Appl. Catal. B-Environ. 2015;166-167:18-24.

23. Harfmann RG, Crouch SR. Kinetic study of Berthelot reaction steps in the absence and presence of coupling reagents. Talanta 1989;36:261-269.

24. Hwang Y, Salatas A, Mines PD, Jakobsen MH, Andersen HR. Graduated characterization method using a multi-well microplate for reducing reactivity of nanoscale zero valent iron materials. Appl. Catal. B-Environ. 2016;181:314-320.

25. Mines PD, Kaarsholm KMS, Droumpali A, Andersen HR, Lee W, Hwang Y. Termination of nanoscale zero-valent iron reactivity by addition of bromate as a reducing reactivity competitor. J. Nanopart. Res. 2017;19:301.

26. McKnight US, Funder SG, Rasmussen JJ, Finkel M, Binning PJ, Bjerg PL. An integrated model for assessing the risk of TCE groundwater contamination to human receptors and surface water ecosystems. Ecol. Eng. 2010;36:1126-1137.

27. Squillace PJ, Thurman EM, Furlong ET. Groundwater as a nonpoint source of atrazine and deethylatrazine in a river during base flow conditions. Water Resour. Res. 1993;29:1719-1729.

28. Wang Q, Snyder S, Kim J, Choi H. Aqueous ethanol modified nanoscale zerovalent iron in bromate reduction: Synthesis, char- 
acterization, and reactivity. Environ. Sci. Technol. 2009;43: 3292-3299.

29. Hansen KMS, Willach S, Antoniou MG, Mosbæk H, Albrechtsen $\mathrm{H}$, Andersen HR. Effect of $\mathrm{pH}$ on the formation of disinfection byproducts in swimming pool water - Is less THM better? Water Res. 2012;46:6399-6409.

30. Wang W, Jin Z, Li T, Zhang H, Gao S. Preparation of spherical iron nanoclusters in ethanol-water solution for nitrate removal. Chemosphere 2006;65:1396-1404.

31. Nadagouda MN, Castle AB, Murdock RC, Hussain SM, Varma RS. In vitro biocompatibility of nanoscale zerovalent iron particles (NZVI) synthesized using tea polyphenols. Green Chem. 2010;12:114-122.

32. Ryu A, Jeong S, Jang A, Choi H. Reduction of highly concentrated nitrate using nanoscale zero-valent iron: Effects of aggregation and catalyst on reactivity. Appl. Catal. B-Environ. 2011;105: 128-135.

33. Tomishige K, Li D, Tamura M, Nakagawa Y. Nickel-iron alloy catalysts for reforming of hydrocarbons: Preparation, structure, and catalytic properties. Catal. Sci. Technol. 2017;7:3952-3979.

34. Ko SO, Lee DH, Kim YH. Kinetic studies of reductive dechlorination of chlorophenols with $\mathrm{Ni} / \mathrm{Fe}$ bimetallic particles. Environ. Technol. 2007;28:583-593.

35. Barnes RJ, Riba O, Gardner MN, Scott TB, Jackman SA, Thompson IP. Optimization of nano-scale nickel/iron particles for the reduction of high concentration chlorinated aliphatic hydrocarbon solutions. Chemosphere 2010;79:448-454.

36. Lai B, Zhang Y, Chen Z, Yang P, Zhou Y, Wang J. Removal of p-nitrophenol (PNP) in aqueous solution by the micron-scale iron-copper $(\mathrm{Fe} / \mathrm{Cu})$ bimetallic particles. Appl. Catal. B-Environ. 2014;144:816-830.

37. Li T, Farrell J. Reductive dechlorination of trichloroethene and carbon tetrachloride using iron and palladized-iron cathodes. Environ. Sci. Technol. 2000;34:173-179.

38. Arnold WA, Roberts AL. Pathways and kinetics of chlorinated ethylene and chlorinated acetylene reaction with $\mathrm{Fe}(0)$ particles. Environ. Sci. Technol. 2000;34:1794-1805. 